\title{
Evaluation of the Behavior of Spinal Deformities in Tuberculosis of the Spine in Adults
}

\author{
Roop Singh, Narender Kumar Magu \\ Department of Orthopaedic Surgery, Paraplegia and Rehabilitation, \\ Pandit Bhagwat Dayal Sharma Post Graduate Institute of Medical Sciences (PGIMS), Rohtak, India
}

\begin{abstract}
Study Design: A prospective clinical study.
Purpose: The objective of the present study was to evaluate the behavior of spinal deformities in tuberculosis (TB) of the spine during the initial 2 years and to suggest remedial measures.

Overview of Literature: Spinal TB is the most common cause of a kyphotic deformity in many parts of the world. Treatment of the established deformity is difficult, hazardous and has a high complication rate.

Methods: We followed 50 adult patients treated for spinal TB for a minimum of 2 years. Average values of vertebral body height loss (VBL), deformity angle, kyphosis angle, and lumbosacral joint angle at the final follow-up were compared with the values at initial presentation. The relationship between the amount of initial VBL and final kyphotic angle was analyzed.

Results: Average values of VBL, deformity angle, kyphosis angle, and lumbosacral joint angle at initial presentation were 0.26, $12.51^{\circ}, 2.26^{\circ}$, and $12.3^{\circ}$, respectively; and the corresponding values at the final follow-up were $0.7,17.8^{\circ}, 5.64^{\circ}$, and $10.8^{\circ}$, respectively. The increase was extremely significant for the deformity angle (initial vs. 6th month, $p=0.000$; 6th month vs. 24th month, $p=0.000$ ) and kyphotic angle (initial vs. 6th month, $p=0.003$; 6th month vs. 24th month, $p=0.000$ ) in the thoracic and thoracolumbar regions during the first 2 years of the disease process. The increase in the deformity angle in the lumbar region was significant only in the initial 6 months $(p=0.01)$. We could not find any correlation between the initial VBL and the final kyphotic angle $(r=0.302, p>0.05)$. Conclusions: Different regions of the vertebral column respond differently to bony destruction caused by spinal TB. Deformity progression is more significant during the initial 6 months of the disease process, and this may be the best time to take remedial measures to prevent development/progression of the deformity. Kyphotic deformity keeps increasing even after 6 months of antituberculous treatment, and it does not correlate with the initial VBL in adults.
\end{abstract}

Keywords: Spinal tuberculosis; Deformity; Vertebrae

\section{Introduction}

The morphologic and pathophysiologic changes encountered in spinal tuberculosis (TB) are the most severe and most varied among infective spondylitis $[1,2]$. TB of the spine demineralizes and destroys the vertebral body, caus- ing pain and deformity (kyphosis) and sometimes spinal cord compression (Pott paraplegia) [3]. In countries where TB is rampant, more than $80 \%$ of the patients with spinal involvement have some sort of detectable kyphosis at the time of presentation. Patients treated conservatively have an average increase of $15^{\circ}$ in deformity and 3\% to 5\%

Received Dec 30, 2014; Revised Feb 4, 2015; Accepted Feb 20, 2015

Corresponding author: Roop Singh

Department of Orthopaedic Surgery, Paraplegia and Rehabilitation, Pandit Bhagwat Dayal Sharma Post Graduate

Institute of Medical Sciences (PGIMS), 52/9-J, Medical Enclave, Rohtak, Haryana 124001, India

Tel: +91-1262-213171, Fax: +91-1262-211308, E-mail: drroopsingh@rediffmail.com 
of the patients end up with a deformity greater than $60^{\circ}$ $[4,5]$. Treatment of the established deformity is difficult, hazardous and has a high complication rate. Kyphosis can be corrected in the active stage of the disease; therefore, early diagnosis and prompt treatment are essential to prevent permanent neurological deficit and/or progression of spinal deformity [6].

In the present study, behavior of the spinal deformity from initial presentation to posttreatment was evaluated at various levels of the vertebral column. It was hypothesized that the different regions of the vertebral column respond differently to bone destruction caused by $\mathrm{TB}$ of the spine.

\section{Materials and Methods}

This prospective study was carried in 50 patients aged more than 15 years (mean age, $42.76 \pm 15.74$ years; range, 17-70 years) and who suffered from TB of the spine from November 2007 to November 2012. There were 30 males $(60 \%)$ and 20 females (40\%). The cervical spine $(n=2)$, dorsal spine $(n=12)$, thoracolumbar junction $(n=7)$, lumbar spine $(n=20)$, and lumbosacral junction $(n=9)$ were affected in patients. A total of 68 consecutive patients were enrolled during this period, but only 50 patients completed full two years of regular follow-up with all relevant investigations as per the study protocol. The diagnosis was confirmed by clinicoradiologic evaluation and by observing response to antitubercular treatment (ATT).

Detailed informative history of the patients was taken. Thorough clinical examination, both physical and neurological examination was performed. Neurological classification was done according to Tuli's classification of stages of paraplegia [7]. Baseline hematological investigations like complete blood count, erythrocyte sedimentation rate (ESR), liver function tests, renal function tests, serum proteins, albumin-globulin ratio, human immunodeficiency virus, and hepatitis $B$ virus surface antigen were carried out.

Radiographic evaluation included plain X-rays of the chest; affected vertebral column; and any other affected part of the body. All lateral radiographs of the spine were taken with the patient standing, and in the lateral decubitus position when the patient was unable to stand (with neurological deficit). Ultrasonography of the cold abscess (paraspinal, psoas) was performed to assess the initial size of the abscess.
In other investigations like fine needle aspiration cytology (if diagnosis was doubtful) and in case of therapeutic drainage of pus, the samples were subjected to ZiehlNeelsen (Z-N) staining and culture sensitivity. In 11 patients who were operated for neurological deterioration, the resected tissue was sent for histopathological examination; and pus and granulation tissue were subjected to Z-N staining and culture sensitivity. Three sputum samples were tested for acid fast bacilli. Tuberculin skin test (Mantoux test) was performed.

After the initial clinical examination and requisite investigations, the treatment protocol was decided. Patients requiring conservative treatment were put on ATT for 12 months. Braces (thoracic lumbar sacral orthosis, taylor brace for lesion from fourth dorsal to second lumbar vertebrae; lumbar sacral orthosis, Goldthwait brace for second lumbar to lumbosacral region) were applied to prevent deformity of the spine. Requisite surgical procedures along with ATT were performed in patients requiring surgery for both neurological involvement and drainage of pus. Follow-up was performed monthly for the first 3 months, 3 monthly for 1 year, and 6 monthly for 2 years. At any time during the follow-up if a patient needed alteration in ATT or surgical intervention, it was carried out. Patients who showed healing of tuberculous lesions on evaluation, both clinically and by investigations (hematologic and radiologic), after 6 months of ATT were the candidates for stopping further therapy. Patients who did not show healing even after 12 months of treatment were considered for appropriate extended ATT. These cases were discussed with the Chest and TB specialists from the institute. Usually, rifampicin and isoniazid were given for the extended period. Complete physical and neurological examinations were performed at each follow-up. Complete blood count and ESR were performed to observe the healing response. Plain X-ray of the area was taken at each follow-up.

Methods used for the assessment of chronological changes in the deformity of the spine were as follows: (1) Deformity angle is formed by the upper and lower borders of the diseased vertebrae, which assesses the deformity, irrespective of the influence of adjacent normal discs and vertebrae [8]. (2) Angle of kyphosis was measured by a technique similar to what described by Konstam and Blesovsky [9]. (3) Lumbosacral joint angle is formed by lines across the cranial border of the fifth lumbar vertebral body and the cranial border of the first sacral vertebral 
body [10]. (4) Assessment of the vertebral body loss (VBL) was done as described by Rajasekaran and Shanmugasundaram [11].

All of the radiological measurements were performed by the first author three times at different occasions and the mean of all three measurements was used for the research purpose. At the end of the study, the data was collected and analyzed by using Student $t$-test, chi-square test, and repeated measures analysis of variance.

\section{Results}

Neurological involvement was seen in 11 patients at the initial presentation (stage II in 2 patients; stage III in 4 patients; and stage IV in 5 patients according to Tuli's classification of paraplegia). Psoas abscesses were found in nine patients, and four of these patients underwent therapeutic drainage of the large abscess. Z-N staining showed acid fast bacilli in three out of these four patients. Histopathological examination of the biopsy material from 11 patients operated for neurological involvement showed classical findings of TB. In all of these patients, culture and sensitivity did not show multidrug resistant $\mathrm{k}$.

Average values of VBL, deformity angle, kyphosis angle, and lumbosacral joint angle at the initial presentation were $0.26,12.51^{\circ}, 2.26^{\circ}$, and $12.3^{\circ}$, respectively; the corresponding values at the final follow-up were $0.7,17.8^{\circ}$, $5.64^{\circ}$, and $10.8^{\circ}$, respectively. Region-wise values are shown in Table 1.

Kyphotic and deformity angles at initial presentation and final follow-up at 24 months were compared between the thoracic and thoracolumbar junction and the lumbar spine. Fifteen patients (78.9\%) showed an increase in the kyphotic angle in the thoracic and thoracolumbar junction area; three patients (15.7\%) showed no change in the kyphotic angle in the thoracic and thoracolumbar junction area, and one patient (5.2\%) showed a decrease in the kyphotic angle in the thoracic and thoracolumbar junction area. In the lumbar spine, eight patients (40\%) showed an increase in the kyphotic angle; five patients (25\%) showed no change in the kyphotic angle, and seven patients (35\%) showed a decrease in the kyphotic angle. Deformity angle at the thoracic and thoracolumbar junction area was increased in 17 patients (89.5\%), and no change in the deformity angle at the thoracic and thoracolumbar junction area was observed in two patients (10.5\%). In the lumbar spine, an increase in the deformity

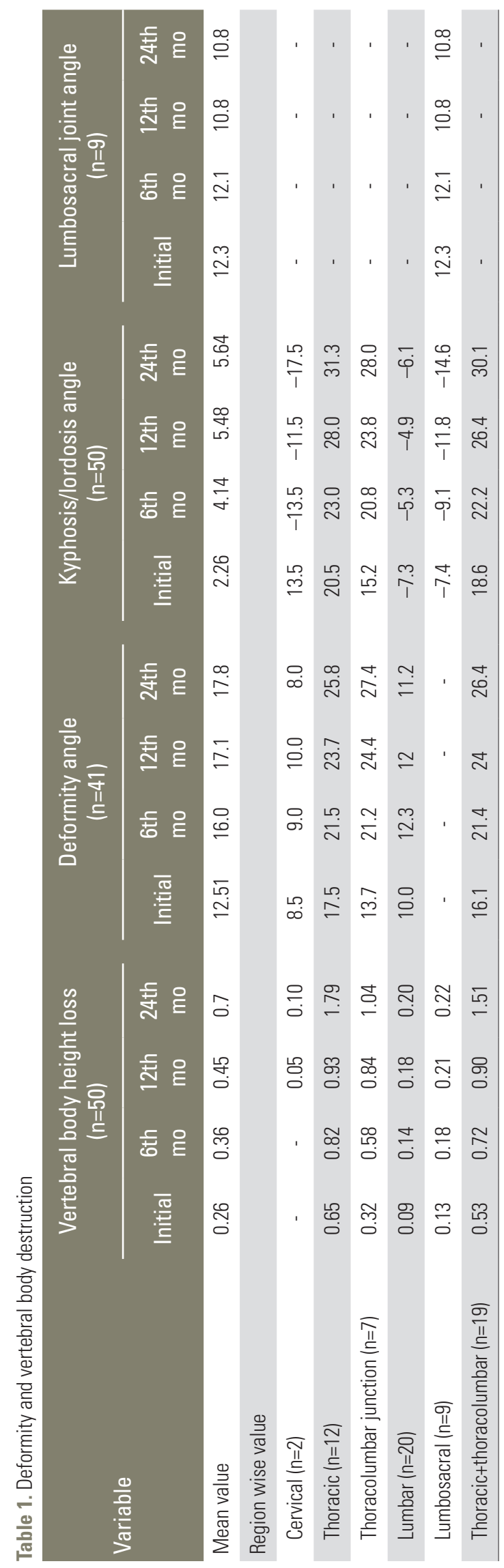


Table 2. Comparison of deformity and kyphotic angles at various stages of the disease

\begin{tabular}{|c|c|c|c|c|c|c|}
\hline \multirow{2}{*}{ Region } & \multirow{2}{*}{ Angle } & \multirow{2}{*}{ Initial } & \multirow{2}{*}{ 6th mo } & \multirow{2}{*}{ 24th mo } & \multicolumn{2}{|c|}{$p$-value } \\
\hline & & & & & Initial vs. 6th mo & 6th vs. 24th mo \\
\hline \multirow[t]{2}{*}{ Thoracic+thoracolumbar junction $(n=19)$} & Deformity & $16.1 \pm 10.1$ & $21.4 \pm 10.9$ & $16.4 \pm 13.5$ & $0.000^{a)}$ & $0.000^{\mathrm{a})}$ \\
\hline & Kyphotic & $18.6 \pm 10.1$ & $22.2 \pm 11.1$ & $30.6 \pm 15.7$ & $0.003^{b)}$ & $0.000^{\mathrm{a})}$ \\
\hline \multirow[t]{2}{*}{ Lumbar (n=20) } & Deformity & $9.5 \pm 6.9$ & $11.7 \pm 8.0$ & $10.7 \pm 9.5$ & $0.01^{\mathrm{cl}}$ & - \\
\hline & Kyphotic & $-7.35 \pm 5.64$ & $-5.35 \pm 12.2$ & $6.1 \pm 12.2$ & - & - \\
\hline
\end{tabular}

Values are presented as mean \pm standard deviation.

a) Extremely significant; ${ }^{\text {b) }}$ Very significant; ${ }^{\text {c) }}$ Significant.

angle was observed in 11 patients (55\%); no change in the deformity angle was observed in four patients (20\%), and a decrease in the deformity angle was observed in five patients (25\%). A comparison of deformity and kyphotic angles at various stages of the disease process and their statistical significance is shown in Table 2.

\section{Discussion}

VBL observed in the present study was the least in cervical and lumbar regions of the vertebral column. This may be due to the biomechanical reasons as lordosis is normally present in these areas. Jain et al. [12] reported a mean VBL of 0.77 (range, 0.1-2.0) in the group treated with ambulant multi-drug chemotherapy (group A) and a mean VBL of 0.67 (range, $0.2-1.5$ ) in patients treated surgically with anterolateral decompression (group B). Initial VBL was found to be less than one in 59 patients (84\%). Rajasekaran and Soundarapandian [13] found an initial VBL of less than one in only $60 \%$ of his patients. In the present study, we found an initial VBL of less than one in $94 \%$ of the cases.

The average initial kyphotic angle observed in the dorsal spine and thoracolumbar junction spine was $18.6^{\circ}$ and $30.1^{\circ}$ at 24 months in the present study. Jain et al. [12] reported a pretreatment angle of $24.3^{\circ}$ (range, $1^{\circ}-73^{\circ}$ ) and a mean observed angle of $37.7^{\circ}$ (range, $8^{\circ}-81^{\circ}$ ) in patients with a single spine lesion at level L2 or above and who were treated with ambulant multidrug treatment; and a mean pretreatment angle of $25.9^{\circ}$ (range, $7^{\circ}-84^{\circ}$ ) and mean observed angles of $26.8^{\circ}$ (range, $3^{\circ}-63^{\circ}$ ) in 30 patients treated by anterolateral decompression. Moon et al. [14] reported average kyphotic angles in the lumbar spine of $4.2^{\circ}, 4.6^{\circ}, 4.4^{\circ}, 2.3^{\circ}, 2.3^{\circ}$, and $2.0^{\circ}$ before treatment and at $3,6,12,18$, and 36 months after treatment, while in the present study, the corresponding values were $-7.3^{\circ}$, $-5.3^{\circ},-4.9^{\circ}$, and $-6.1^{\circ}$ at initial presentation and at 6,12 , and 24 months, respectively. In the present study, normal lumbar lordosis was comparatively maintained. Moon et al. [14] reported that the average deformity angles in the lumbar spine before treatment and at $3,6,12,18$, and 36 months after treatment were $6.2^{\circ}, 7.2^{\circ}, 7.9^{\circ}, 8.2^{\circ}, 8.4^{\circ}$, and $8.6^{\circ}$, respectively. These values were less compared to the values observed in the lumbar spine in the present study. There was an increase in the deformity angle at various sites of the vertebral column in the present study except at the cervical spine at subsequent follow-ups. A mean increase in deformity angles of $8.3^{\circ}, 13.7^{\circ}$, and $1.2^{\circ}$ were observed in the dorsal, thoracolumbar, and lumbar spine, respectively. Tuli $[3,15]$ reported an increase in kyphosis in $67 \%$ of dorsolumbar and $55 \%$ of dorsal lesions. The Medical Research Council in its third report found a mean increase of $6^{\circ}$ in the group with ambulant chemotherapy [16]. Once the diagnosis of TB spine is made and the patient is put on ambulant chemotherapy, kyphosis continues to grow despite being treated. However, the progression of kyphosis can be minimized by prescribing suitable braces [17]. Patients treated nonoperatively have an average increase of $15^{\circ}$ in deformity and $3 \%$ to $5 \%$ of patients end up with a deformity greater than $60^{\circ}[3,11]$. Kyphosis, once healed, with osseous fusion does not grow alarmingly in adults in later life. However, when the lesion heals with fibrous or fibro-osseous healing, it may progress further [17].

Similar to the observation by Jain et al. [12], the kyphotic angle achieved at 6 months was not maintained at the 24-month follow-up in the present series, also. Fifteen patients (78.9\%) showed an increase in the kyphotic angle 
in the thoracic and thoracolumbar junction area, $17 \mathrm{pa}$ tients (89.5\%) showed an increase in the deformity angle. The increase in the deformity angle was extremely significant between initial presentation and 6 months $(p=0.000)$; 6 months and 24 months $(p=0.000)$. Increase in the kyphotic angle was also statistically significant between initial presentation and 6 months ( $p=0.003)$; and 6 months and 24 months $(p=0.000)$. Upadhyay et al. [18] reported a statistically significant difference only in preoperative values and 6 month $(p=0.001)$ values of the kyphotic angle between radical and debridement groups in these areas of the spine. There was no statistically significant difference in other periods of follow-up till 5 years. Deformity angle also showed a similar pattern of results.

In the lumbar spine, only eight patients (40\%) showed an increase in the kyphotic angle, and 11 patients (55\%) showed an increase in the deformity angle. This increase in the kyphotic angle was not statistically significant between initial presentation and 6 months and 24 months. Increase in the deformity angle was significant $(p<0.01)$ only between initial presentation and 6 months. Upadhyay et al. [18] reported a significant difference in both kyphotic angle $(p=0.001)$ and deformity angle $(p<0.001)$ between radical and debridement groups in preoperative and 6-month values. Similar to the observations of the present study, they also did not find any statistically significant difference subsequently. Rajasekaran et al. [19] reported that the average pretreatment kyphotic angle in the lumbosacral area was $6.4^{\circ}$ and it increased to $10.2^{\circ}$ at the end of 15 years. Even though the tendency for kyphosis is less in this area, most patients end up with gross hypolordosis or straightening of the lumbar spine. Only four patients (7\%) maintained lordosis, and 31 patients (58\%) had complete straightening of the spine [19].

As reported by Moon et al. [14], we also observed that the pattern of vertebral body collapse in the lumbar spine was different from that in the thoracic spine, and that minimal kyphosis developed as a sequel at the time of healing of TB. Rajasekaran et al. [19] reported that the presence of normal lumbar lordosis seemed to alter the pattern of collapse. In the dorsal and dorsolumbar lesion with vertebral destruction, acute kyphosis developed until there was contact with healthy vertebrae. Generally in the past, it was considered that the pattern of vertebral body collapse in spinal TB is the wedge-shaped type, which leads to kyphosis of the spine. However, it was found that the pattern of the vertebral body collapse in TB was verti- cal (telescoping) in patients who were treated $[4,19]$.

Puig Guri [20] stated that the collapse in TB spondylitis can occur along a longitudinal axis (telescopy) or by flexion in the sagittal plane of one spinal segment on the other (inflexion). The destruction of a thoracic vertebral body results in posterior displacement of the center of motion, subluxation at the level of the facets, and an increase in the weight to be borne by the anterior part of the body. The horizontal orientation of the articular facets and pre-existing kyphotic curve of the dorsal spine aids gravity in progression of the deformity. In the lumbar region, because of the larger size of the disc, the vertical position of the articular facets, and the relative narrowness of the pedicle, a marked amount of telescoping was possible [20].

It was also previously reported that the patients who had a lesion of the thoracic spine had more progression in the angle of kyphosis than did those who had a thoracolumbar lesion, with the least increase occurring in patients who had a lumbar or a lumbosacral lesion [4,13,14,18-23].

In the literature it was observed that the initial VBL and the final kyphotic angle showed a correlation [11]. In the present study, we could not find such a correlation $(r=0.302, p>0.05)$. Jain et al. [12] reported that in the conservative group, the initial VBL and the observed final angle had better correlation than in the operative group.

The average lumbosacral joint angles observed were $12.3^{\circ}, 12.1^{\circ}, 10.8^{\circ}$, and $10.8^{\circ}$ at initial presentation, and at 6,12 , and 24 months, respectively in the present study. Moon et al. [14] reported lumbosacral joint angles measuring $32^{\circ}, 30^{\circ}, 28^{\circ}, 26^{\circ}, 26^{\circ}$, and $26^{\circ}$ at pretreatment and $3,6,12,18$, and 36 months after treatment. In both the studies, lumbosacral joint angles did not show any change after 12 months.

Moon et al. [14] reported that patients with TB of the lumbosacral joint could also be treated successfully with minimal changes in the lumbosacral joint angle. Even in patients with a decreased lumbosacral joint angle with or without fusion (secondary sacralisation of L5), an upper normal joint could compensate for the loss of lumbosacral joint motion by developing hyperlordosis. Rajasekaran et al. [19] reported the phenomenon of subsidence in lower lumbar lesions, in which the superior vertebra gradually descended without kyphosis. Here the inferior surface of the superior vertebra and the superior surface of the inferior vertebra came into contact with each other, with loss of normal lordosis but without kyphosis. 
The present study has a few limitations. We did not separately analyze the data for surgically treated and conservatively treated patients. There were fewer patients with TB in some regions of the vertebral column like the cervical spine.

\section{Conclusions}

Different regions of the vertebral column respond differently to bony destruction caused by spinal TB. Deformity progression is more significant during the initial 6 months of the disease process, and this may be the best time to take remedial measures to prevent development/progression of the deformity. Kyphotic deformity keeps increasing even after 6 months of antituberculous treatment, and it does not correlate with the initial VBL in adults.

\section{Conflict of Interest}

No potential conflict of interest relevant to this article was reported.

\section{References}

1. Hoffman EB, Crosier JH, Cremin BJ. Imaging in children with spinal tuberculosis: a comparison of radiography, computed tomography and magnetic resonance imaging. J Bone Joint Surg Br 1993;75:2339.

2. Agarwal S, Jain UK. Management of spinal tuberculosis: current concepts. J Indian Med Assoc 2004;102: 164-7.

3. Tuli SM. Severe kyphotic deformity in tuberculosis of the spine. Int Orthop 1995;19:327-31.

4. Moon MS, Lee MK. The changes of the kyphosis of the tuberculous spine in children following ambulant treatment. J Korean Orthop Assoc 1971;6:203-8.

5. Parthasarathy R, Sriram K, Santha T, Prabhakar R, Somasundaram PR, Sivasubramanian S. Shortcourse chemotherapy for tuberculosis of the spine. A comparison between ambulant treatment and radical surgery: ten-year report. J Bone Joint Surg Br 1999; 81:464-71.

6. Jain AK. Treatment of tuberculosis of the spine with neurologic complications. Clin Orthop Relat Res 2002;(398):75-84.

7. Tuli SM. Judicious management of tuberculosis of bones, joints and spine. Indian J Orthop 1985;19:14766.

8. Tull SM, Kumar S. Early results of treatment of spinal tuberculosis by triple drug therapy. Clin Orthop Relat Res 1971;81:56-70.

9. Konstam PG, Blesovsky A. The ambulant treatment of spinal tuberculosis. Br J Surg 1962;50:26-38.

10. Moon MS, Woo YK, Lee KS, Ha KY, Kim SS, Sun $\mathrm{DH}$. Posterior instrumentation and anterior interbody fusion for tuberculous kyphosis of dorsal and lumbar spines. Spine (Phila Pa 1976) 1995;20:1910-6.

11. Rajasekaran S, Shanmugasundaram TK. Prediction of the angle of gibbus deformity in tuberculosis of the spine. J Bone Joint Surg Am 1987;69:503-9.

12. Jain AK, Aggarwal PK, Arora A, Singh S. Behaviour of the kyphotic angle in spinal tuberculosis. Int Orthop 2004;28:110-4.

13. Rajasekaran S, Soundarapandian S. Progression of kyphosis in tuberculosis of the spine treated by anterior arthrodesis. J Bone Joint Surg Am 1989;71:131423.

14. Moon MS, Moon YW, Moon JL, Kim SS, Sun DH. Conservative treatment of tuberculosis of the lumbar and lumbosacral spine. Clin Orthop Relat Res 2002;(398):40-9.

15. Tuli SM. Results of treatment of spinal tuberculosis by "middle-path" regime. J Bone Joint Surg Br 1975;57:13-23.

16. A controlled trial of debridement and ambulatory treatment in the management of tuberculosis of the spine in patients on standard chemotherapy: a study in Bulawayo, Rhodesia. J Trop Med Hyg 1974;77:7292.

17. Jain AK, Dhammi IK, Jain S, Mishra P. Kyphosis in spinal tuberculosis: prevention and correction. Indian J Orthop 2010;44:127-36.

18. Upadhyay SS, Saji MJ, Sell P, Sell B, Hsu LC. Spinal deformity after childhood surgery for tuberculosis of the spine: a comparison of radical surgery and debridement. J Bone Joint Surg Br 1994;76:91-8.

19. Rajasekaran S, Shanmugasundaram TK, Prabhakar R, Dheenadhayalan J, Shetty AP, Shetty DK. Tuberculous lesions of the lumbosacral region: a 15 -year follow-up of patients treated by ambulant chemotherapy. Spine (Phila Pa 1976) 1998;23:1163-7.

20. Puig Guri J. The formation and significance of vertebral ankylosis in tuberculous spines. J Bone Joint 
Surg Am 1947;29:136-48.

21. A 15-year assessment of controlled trials of the management of tuberculosis of the spine in Korea and Hong Kong: thirteenth report of the medical research Council Working Party on tuberculosis of the spine. J Bone Joint Surg Br 1998;80:456-62.

22. Controlled trial of short-course regimens of chemotherapy in the ambulatory treatment of spinal tuberculosis. Results at three years of a study in Korea: twelfth report of the Medical Research Council
Working Party on tuberculosis of the spine. J Bone Joint Surg Br 1993;75:240-8.

23. Five-year assessment of controlled trials of shortcourse chemotherapy regimens of 6,9 or 18 months' duration for spinal tuberculosis in patients ambulatory from the start or undergoing radical surgery: fourteenth report of the Medical Research Council Working Party on tuberculosis of the spine. Int Orthop 1999;23:73-81. 